\title{
Perioperative neutrophil to lymphocyte ratio as a predictor of poor cardiac surgery patient outcomes
}

This article was published in the following Dove Press journal:

Pragmatic and Observational Research

15 February 2017

Number of times this article has been viewed

\author{
Konstantinos \\ Giakoumidakis' \\ Nikolaos V Fotos ${ }^{2}$ \\ Athina Patelarou ${ }^{3}$ \\ Stavros Theologou' \\ Mihalis Argiriou ${ }^{4}$ \\ Anastasia A Chatziefstratiou ${ }^{2}$ \\ Christina Katzilieri ${ }^{5}$ \\ Hero Brokalaki² \\ 'Cardiac Surgery Intensive Care Unit, \\ "Evangelismos" General Hospital \\ of Athens, 'Faculty of Nursing, \\ School of Healthcare Sciences, \\ National \& Kapodistrian University \\ of Athens, Athens, ${ }^{3}$ Department of \\ Anaesthesiology, University Hospital \\ of Heraklion, Heraklion, ${ }^{4}$ Department \\ of Cardiothoracic Surgery, \\ "Evangelismos" General Hospital \\ of Athens, ${ }^{5}$ Intensive Care Unit, \\ "Korgalenio - Benakio Hellenic Red \\ Cross" General Hospital of Athens, \\ Athens, Greece
}

Purpose: The purpose of the present study was to investigate the association between the perioperative neutrophil to lymphocyte ratio (NLR) and cardiac surgery patient outcomes.

Patients and methods: A retrospective cohort study of 145 patients who underwent cardiac surgery in a tertiary hospital of Athens, Greece, from January to March 2015, was conducted. By using a structured short questionnaire, this study reviewed the electronic hospital database and the medical and nursing patient records for data collection purposes. The statistical significance was two-tailed, and $p$-values $<0.05$ were considered significant. The statistical analysis was performed with Mann-Whitney $U$ test and Spearman's correlation coefficient, by using the Statistical Package for Social Sciences software (IBM SPSS 21.0 for Windows).

Results: The increased preoperative levels of NLR were associated with significantly higher mortality, both in-hospital ( $p=0.001)$ and 30-day ( $p=0.002)$, prolonged postoperative hospital length of stay (LOS), both in the cardiac intensive care unit (ICU) ( $p=0.002)$, and in-hospital $(p=0.018)$, and likewise with delayed tracheal extubation $(p \leq 0.001)$. Furthermore, patients with elevated NLR during the second postoperative day had significantly higher in-hospital mortality ( $p=0.018)$, increased incidence of pneumonia $(p=0.022)$, higher probability of readmission to the ICU ( $p=0.002)$, prolonged ICU LOS ( $p \leq 0.001)$, and delayed tracheal extubation ( $p \leq 0.001)$. Conclusion: Increased perioperative NLR seems to be associated with significantly higher mortality and morbidity in cardiac surgery patients. At the same time, NLR is a significant and inexpensive biomarker for the early identification of patients at high risk for complications. In addition, NLR levels could lead clinicians to perform measures for the optimal therapeutic patient approach.

Keywords: airway extubation, hospital mortality, intensive care units, length of stay, leukocytes, patient readmission, pneumonia

\section{Introduction}

Although increased white cell counts are general markers of the inflammatory response to various stimuli and have been used as indicators of morbidity and mortality of patients with cardiovascular diseases, ${ }^{1,2}$ their predictive value seems to be significantly low. ${ }^{3}$ Apart from the total number of white blood cells, various white cell subtypes, such as neutrophils and lymphocytes, have an active role in the inflammatory process of chronic cardiovascular disease. ${ }^{4,5}$ Besides, raised neutrophil and decreased lymphocyte counts have been associated with poor outcomes in patients with acute coronary syndrome or ischemic stroke. ${ }^{6}$

The neutrophil to lymphocyte ratio (NLR) is a well-studied biomarker of the patients' inflammatory response, ${ }^{7,8}$ especially in those with surgical treatment of a malignancy. ${ }^{9,10}$ Moreover, this indicator has been investigated in patients with
Correspondence: Konstantinos Giakoumidakis

Cardiac Surgery Intensive Care Unit,

"Evangelismos" General Hospital of

Athens, 45-47 Ipsilantou Street, 10676,

Athens, Greece

Tel +306973793489

Fax +302132041987

Email kongiakoumidakis@gmail.com 
cardiovascular disease ${ }^{11-13}$ and it has been observed that the number of white cell counts or any type of leukocyte separately has greater ability to predict mortality and morbidity in patients undergoing coronary angiography and angioplasty, or even those with acute myocardial infarction (AMI) or chronic coronary disease. ${ }^{14,15}$ However, the NLR has been assessed in patients undergoing cardiac surgical procedures, which are characterized by significant systemic inflammation response due to the surgical procedure and the application of cardiopulmonary bypass. ${ }^{16}$

The purpose of this study was to investigate the association between perioperative NLR and patient outcomes after open heart surgery. This study intended to add new data to the important literature and research deficit on the effect of NLR on cardiac surgery patient outcomes. In addition, the possible association of NLR with the patient outcomes will allow the early identification of high-risk patients, through a low-cost biomarker and could contribute to clinical decisions and measures for the prevention of complications, achieving the optimal health care for these patients.

\section{Materials and methods}

\section{Study design, variables, and participants}

A retrospective cohort study was conducted. The perioperative NLR, as measured at three different times, was the independent variable of the present study. Specifically, NLR was measured preoperatively (within $24 \mathrm{~h}$ before the beginning of the surgical operation), during the first and the second postoperative days. The dependent variables (outcomes) of this study were the following: 30-day mortality, in-hospital mortality, cardiac surgery intensive care unit length of stay (ICU LOS), postoperative LOS, surgical re-exploration due to mediastinal bleeding, occurrence of nosocomial pneumonia, patient readmission to the cardiac surgery ICU, and the time of tracheal extubation.

The inclusion criteria of this study were a priori defined as follows: 1) age $\geq 18$ years, and 2 ) open-heart surgery. The study excluded patients with recent AMI (within the last 7 days before the surgery), those with known hematologic malignancy, patients without preoperative and postoperative measurement of neutrophil and leukocyte levels in their blood, those with the absence of socio-demographic and clinical data in their medical and nursing records, and finally patients who were admitted to the cardiac surgery ICU for medical reasons, other than open-heart surgery, such as those with acute coronary syndrome without surgical treatment and those who underwent cardiac catheterization for diagnostic reasons.

\section{Methods and data collection}

Data collection was performed retrospectively in December 2015. For data collection purposes, preoperative and postoperative levels of neutrophils and lymphocytes (first and second postoperative days) were obtained through the electronic database of the hospital. NLR was calculated on the basis of these levels. Socio-demographic and clinical patient data were collected by using a structured short questionnaire and through medical and nursing patient record review, which was conducted by one of the researchers simultaneously, aiming to ensure the reliability and validity of the data collection process.

\section{Statistical analysis}

Categorical and numerical data are expressed as n (\%) and mean ( \pm standard deviation $[\mathrm{SD}]$ ), respectively. Bivariate analyses were performed by using the Kruskal-Wallis test to examine the possible association between numerical and categorical variables and the Spearman's correlation coefficient to examine the possible association between numerical variables. All tests of statistical significance were two-tailed, and $p$-values $<0.05$ were considered statistically significant. Analyses were conducted using IBM SPSS 21.0 for Windows.

\section{Ethics}

The data collection was conducted after obtaining written permission from the ethics committee of the "Evangelismos" General Hospital of Athens, Greece. The requirement of patient consent was waived by the hospital ethics committee as the data collection was carried out retrospectively. The investigation was carried out in accordance with the ethical standards of the responsible institutional committee for human experimentation and with the Helsinki Declaration of 1975, as revised in 2013. Precaution measures were taken in order to protect the privacy of the research subjects and the confidentiality of their personal information, including limiting the amount of personal information to the absolute minimum, assigning an identification number to each subject and attaching the identification number to the actual research information, removing the subject names as soon as data were analyzed and maintaining any identifying information and lists of identification numbers in a safe and locked file.

\section{Results}

During a 3-month period, from January 2015 to March 2015, 204 patients were admitted to the eight-bed cardiac surgery ICU of a tertiary hospital of Athens, Greece and were eligible for enrollment in the present study. Based on the aforementioned 
inclusion and exclusion criteria, 145 patients (participation rate $71.1 \%$ ) constituted the final study sample. The mean age of patients in this study was 65.5 years, while the majority of them were males (68.3\%). Approximately half of the patients underwent exclusively coronary artery bypass grafting $(46.2 \%)$, while emergency heart surgery was performed in only 13 patients (9\%). Table 1 summarizes the socio-demographic, clinical, and perioperative patient characteristics.

The mean perioperative (preoperative, first, and second postoperative day) NLR was $4.2,14.5$, and 12.1 , respectively (Table 2). Table 3 presents the main outcomes of patients in this study. The mean ICU and in-hospital LOS were 6.4 and 13.1 days, respectively, while the 30-day and in-hospital mortality reached $13.1 \%$ and $16.6 \%$, respectively. The incidence of nosocomial pneumonia and readmission to the cardiac ICU was $9.7 \%$ and $3.4 \%$, respectively. Also, the mean time of tracheal intubation was $57.3 \mathrm{~h}$.

Table I Demographic, clinical, and perioperative patient characteristics

\begin{tabular}{|c|c|}
\hline Characteristics & Mean ( \pm SD) \\
\hline Age (years) & $65.5(12)$ \\
\hline BMI $\left(\mathrm{kg} / \mathrm{m}^{2}\right)$ & $27.9(4.6)$ \\
\hline Ejection fraction (\%) & $5 I .3(I I)$ \\
\hline Duration of surgery $(\mathrm{min})$ & $267.1(97.9)$ \\
\hline Duration of cardiopulmonary bypass (min) & $129(77.9)$ \\
\hline \multirow[t]{2}{*}{ EuroSCORE (\%) } & $9.4(11.1)$ \\
\hline & n (\%) \\
\hline \multicolumn{2}{|l|}{ Gender } \\
\hline Male & $99(68.3)$ \\
\hline Female & $46(31.7)$ \\
\hline COPD & $26(17.9)$ \\
\hline DM & $49(33.8)$ \\
\hline CRF & II (7.6) \\
\hline Cardiopulmonary bypass & $129(89)$ \\
\hline \multicolumn{2}{|l|}{ Type of surgery } \\
\hline CABG & $67(46.2)$ \\
\hline Not isolated CABG & $78(53.8)$ \\
\hline \multicolumn{2}{|l|}{ Surgical operation } \\
\hline Emergency & $13(9)$ \\
\hline Elective & $132(9 \mid)$ \\
\hline
\end{tabular}

Abbreviations: $\mathrm{BMI}$, body mass index; SD, standard deviation; COPD, chronic obstructive pulmonary disease; DM, diabetes mellitus; CRF, chronic renal failure; CABG, coronary artery bypass grafting.

Table 2 Perioperative neutrophil to lymphocyte ratio

\begin{tabular}{ll}
\hline NLR & Mean (士SD) \\
\hline Preoperatively & $4.21(10)$ \\
First postoperative day & $14.5(10.1)$ \\
Second postoperative day & $12.1(7.5)$ \\
\hline Abbreviations: SD, standard deviation; NLR, neutrophil to lymphocyte ratio.
\end{tabular}

Table 3 Patient outcomes

\begin{tabular}{ll}
\hline Outcome & Mean ( \pm SD) \\
\hline ICU length of stay (days) & $6.4(15.9)$ \\
Total postoperative length of stay (days) & $13.1(16.8)$ \\
Time until tracheal extubation $(\mathrm{h})$ & $57.3(323)$ \\
\hline & $\mathbf{n}(\%)$ \\
\hline Surgical re-exploration due to mediastinal bleeding & $4(2.8)$ \\
Nosocomial pneumonia & $14(9.7)$ \\
ICU readmission & $5(3.4)$ \\
30-day mortality & $19(13.1)$ \\
In-hospital mortality & $24(16.6)$ \\
\hline
\end{tabular}

Abbreviations: SD, standard deviation; ICU, intensive care unit.

Tables 4 and 5 show the associations of preoperative and second postoperative day NLR with the dependent variables of this study, respectively. Specifically, patients who died within the first 30 days after the surgery demonstrated significantly higher preoperative NLR (3.8 vs $2.0, p=0.002$ ), and there was the same finding for those who died during their hospitalization ( 3.6 vs $2.1, p=0.001$ ). Furthermore, the increased preoperative NLR levels were strongly associated with poor outcomes, such as the prolonged postoperative LOS, either in the ICU $(p=0.002)$ or in-hospital $(p=0.018)$, and the delayed tracheal extubation $(p \leq 0.001)$ (Table 4$)$.

Table 4 Associations between preoperative NLR and dependent variables of the study

\begin{tabular}{|c|c|c|}
\hline Variables & $\begin{array}{l}\text { Median preoperative } \\
\text { NLR }\end{array}$ & $p$-value \\
\hline $\begin{array}{l}\text { Surgical re-exploration due to } \\
\text { mediastinal bleeding }\end{array}$ & & $0.68 I^{a}$ \\
\hline Yes & 2.4 & \\
\hline No & 2.2 & \\
\hline 30-day mortality & & $0.002^{\mathrm{a}}$ \\
\hline Yes & 3.8 & \\
\hline No & 2.0 & \\
\hline In-hospital mortality & & $0.00 I^{\mathrm{a}}$ \\
\hline Yes & 3.6 & \\
\hline No & 2.1 & \\
\hline Nosocomial pneumonia & & $0.199^{a}$ \\
\hline Yes & 3.0 & \\
\hline No & 2.1 & \\
\hline ICU readmission & & $0.085^{\mathrm{a}}$ \\
\hline Yes & 3.7 & \\
\hline No & 2.2 & \\
\hline \multicolumn{3}{|c|}{ Spearman's correlation coefficient } \\
\hline ICU length of stay & 0.251 & $0.002^{b}$ \\
\hline $\begin{array}{l}\text { Total postoperative length } \\
\text { of stay }\end{array}$ & 0.196 & $0.018^{b}$ \\
\hline Time until tracheal extubation & 0.363 & $\leq 0.00 \mathrm{I}^{\mathrm{b}}$ \\
\hline
\end{tabular}

Pragmatic and Observational Research 2017:8

submit your manuscript | www.dovepress.com 
Table 5 Associations between NLR at second postoperative day and dependent variables of the study

\begin{tabular}{|c|c|c|}
\hline Variables & $\begin{array}{l}\text { Median NLR at second } \\
\text { postoperative day }\end{array}$ & p-value \\
\hline $\begin{array}{l}\text { Surgical re-exploration due to } \\
\text { mediastinal bleeding }\end{array}$ & & $0.433^{\mathrm{a}}$ \\
\hline Yes & 17.6 & \\
\hline No & 9.7 & \\
\hline 30-day mortality & & $0.074^{\mathrm{a}}$ \\
\hline Yes & 13.7 & \\
\hline No & 9.6 & \\
\hline In-hospital mortality & & $0.018^{a}$ \\
\hline Yes & 14.0 & \\
\hline No & 9.3 & \\
\hline Nosocomial pneumonia & & $0.022^{a}$ \\
\hline Yes & 13.9 & \\
\hline No & 9.5 & \\
\hline ICU readmission & & $0.002^{\mathrm{a}}$ \\
\hline Yes & 23.7 & \\
\hline No & 9.6 & \\
\hline \multicolumn{3}{|c|}{ Spearman's correlation coefficient } \\
\hline ICU length of stay & 0.316 & $\leq 0.00 \mathrm{I}^{\mathrm{b}}$ \\
\hline $\begin{array}{l}\text { Total postoperative length } \\
\text { of stay }\end{array}$ & 0.142 & $0.088^{\mathrm{b}}$ \\
\hline Time until tracheal extubation & 0.311 & $\leq 0.00 \mathrm{I}^{\mathrm{b}}$ \\
\hline
\end{tabular}

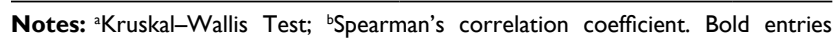
denote $p<0.05$.

Abbreviations: ICU, intensive care unit; NLR, neutrophil-lymphocyte ratio.

Patients who died in-hospital were characterized by significantly higher levels of NLR during the second postoperative day (14.0 vs 9.3, $p=0.018$ ) (Table 5). Likewise, patients who developed nosocomial pneumonia and those who were readmitted to the cardiac ICU exhibited significantly higher NLR during the second postoperative day ( 13.9 vs $9.5, p=0.022$ and 23.7 vs $9.6, p=0.002$, respectively) (Table 5). Finally, significant associations of NLR at the second postoperative day with prolonged ICU stay $(p \leq 0.001)$ and delayed tracheal extubation $(p \leq 0.001)$ are presented in Table 5. Statistically significant relationships between the first postoperative day NLR levels and the investigated patient outcomes were not determined.

\section{Discussion}

The most important finding of this study was the association of the increased perioperative NLR levels with adverse outcomes of patients undergoing cardiac surgery. Specifically, the elevated NLR levels, both preoperatively and at the second postoperative day were associated with significantly higher mortality, prolonged postoperative hospital stay, and delayed tracheal extubation. Furthermore, the increased NLR at the second postoperative day was associated with significantly higher risk of both postoperative pneumonia and patient ICU readmission.
Several investigators have previously attempted to correlate the NLR with the mortality rates of patients with cardiovascular disease and have stated that NLR is a major determinant of mortality among those with chronic coronary disease, AMI, and after coronary angioplasty. ${ }^{17-19}$ However, only two original studies ${ }^{14,20}$ and one meta-analysis ${ }^{4}$ found in the literature review, had investigated the association of perioperative NLR levels with the outcomes of patients undergoing cardiac surgery.

Gibson et $\mathrm{al}^{20}$ in their prospective study of 1,938 coronary artery bypass grafting patients, in line with the findings of the present study, concluded that preoperative NLR was an important independent risk factor for increased patient mortality. Specifically, the increase of NLR by one unit was associated with an increase of mortality by $9 \%$ (HR $1.09,95 \%$ CI: $1.03-1.16, p=0.004)$. Contrary to the findings of this study, the authors reported that patients with raised NLR levels were significantly more likely to require tracheal re-intubation after surgery (odds ratio [OR] 6.29, 95\% confidence interval [CI]: $1.85-21.4, p=0.003$ ), and they had higher cardiac troponin I levels at $24 \mathrm{~h}$ after the surgical procedure. ${ }^{20}$ This study did not examine the association of postoperative NLR with the aforementioned patient outcomes.

The second relevant study was performed by Gibson et $\mathrm{al},{ }^{14}$ in which the authors investigated the possible effect of perioperative (preoperative and at the second postoperative day) NLR levels on the incidence of postoperative atrial fibrillation in 275 cardiac surgery patients. Although this outcome seemed to be strongly associated with the elevated perioperative NLR levels, it was not assessed in this study. However, it remains indicative of both the higher morbidity of patients with increased perioperative NLR and the close relationship of NLR with cellular inflammatory response and oxidative stress, which are the main causes of atrial fibrillation in patients after coronary artery bypass grafting. ${ }^{21}$

The findings of a recent meta-analysis of five studies with a total sample of 3,108 patients after cardiac or vascular surgery are noteworthy. ${ }^{4}$ In line with the present study, the increased preoperative NLR was an important independent predictor of mortality from all causes, since its increase by one unit was strongly associated with higher mortality by $85 \%$ (hazards ratio [HR] 1.85, 95\% CI: $1.46-2.36, p<0.001$ ). On the other hand and in contrast with the findings of this study, the aforementioned meta-analysis investigated the long-term mortality ( $>6$ months) and not the early (within 30 days after surgery) or in-hospital one.

It is worth mentioning that in the currently available published research, we did not find data suggesting that increased 
NLR levels are associated with specific poor patient outcomes, such as the prolonged postoperative in-hospital LOS, the readmission to the cardiac surgery ICU, and the delayed tracheal extubation. Consequently, the findings of the present study on the aforementioned outcomes are of particular interest and highlight the need for further future research.

Interpreting the results of the present study, elevated preoperative NLR may reflect a chronic background inflammatory condition, due to a chronic cardiovascular disease, and probably indicates patients of high cardiovascular risk considering that neutrophilia is often associated with states of hyper-coagulability and unstable atheromatous plaque in coronary disease. ${ }^{22}$ Moreover, the high postoperative NLR highlights not only the degree of myocardial damage due to surgery but also the level of tissue hypoperfusion due to both the low cardiac output and the ischemia-reperfusion syndromes, which frequently follow major operations, including open heart surgery. ${ }^{23}$

In addition, from the available evidence, it is found that the harmful effect of elevated NLR levels on the outcomes of cardiac surgical patients is very likely to be a reflection of pathophysiological disorders resulting from the increased neutrophil count and through the protective role of lymphocytes. Neutrophilia represents low-grade ${ }^{5}$ to unrestrained cellular inflammation, while lymphopenia is indicative of latent immune response. ${ }^{4}$ Significant neutrophilia is observed in acute inflammatory response to tissue injury and also has been associated with reperfusion injury. ${ }^{23}$ In addition, the significant association of neutrophilia with the increased levels of the inflammatory mediators, such as tumor necrosis factor-alpha (TNF- $\alpha$ ) and the interleukins 6, 7, 8, 12, and 17, that are wellknown markers of poor outcomes, such as higher mortality, multiorgan failure, and increased incidence of recurrent ischemic events, in critically ill patients, cannot be overlooked. ${ }^{24,25}$ On the other hand, lymphopenia occurs in cases of increased cortisol production and neuroendocrine stress, while normal and/or elevated lymphocyte count indicates better survival rates in patients with coronary artery disease, heart failure, and those undergoing percutaneous coronary angioplasty. ${ }^{4}$

\section{Study limitations}

To the best of our knowledge, the present study was the first one in Greece to investigate the association between the perioperative NLR levels and the outcomes of patients undergoing cardiac surgery. Moreover, this was one of the few relevant studies worldwide, aiming to add new data to the currently known body of knowledge in a research area that has not been fully investigated. However, the retrospective design of the present study, small sample size, and the fact that the data were collected from a single cardiac surgery center are limitations that threaten the validity of the study and the generalization of the findings to the general population of cardiac surgery patients. These limitations did not allow us to focus on multivariate adjustments. Future research with greater sample size, prospective design, multicenter data collection, and multivariate adjustments is needed.

\section{Conclusion}

Elevated perioperative NLR seems to be associated with significantly higher patient morbidity and mortality rates. Simultaneously, NLR is a reliable and inexpensive biomarker, contributing to the early identification of patients at high risk for complications. In addition, through the use of NLR, clinicians could implement measures for the optimal therapeutic approach of cardiac surgery patients and the elimination of adverse patient outcomes.

\section{Disclosure}

The authors report no conflicts of interest in this work.

\section{References}

1. Bagger JP, Zindrou D, Taylor KM. Leukocyte count: a risk factor for coronary artery bypass graft mortality. Am J Med. 2003;115(8): 660-663.

2. Newall N, Grayson AD, Oo AY, et al. Preoperative white blood cell count is independently associated with higher perioperative cardiac enzyme release and increased 1-year mortality after coronary artery bypass grafting. Ann Thorac Surg. 2006;81(2):583-589.

3. Coller BS. Leukocytosis and ischemic vascular disease morbidity and mortality: is it time to intervene? Arterioscler Thromb Vasc Biol. 2005;25(4):658-670.

4. Tan TP, Arekapudi A, Metha J, Prasad A, Venkatraghavan L. Neutrophillymphocyte ratio as predictor of mortality and morbidity in cardiovascular surgery: a systematic review. ANZ J Surg. 2015;85(6):414-419.

5. Compte N, Bailly B, De Breucker S, Goriely S, Pepersack T. Study of the association of total and differential white blood cell counts with geriatric conditions, cardio-vascular diseases, seric IL-6 levels and telomere length. Exp Gerontol. 2015;61:105-112.

6. Guasti L, Dentali F, Castiglioni L, et al. Neutrophils and clinical outcomes in patients with acute coronary syndromes and/or cardiac revascularisation. A systematic review on more than 34,000 subjects Thromb Haemost. 2011;106(4):591-599.

7. Venkatraghavan L, Tan TP, Mehta J, Arekapudi A, Govindarajulu A, Siu E. Neutrophil lymphocyte ratio as a predictor of systemic inflammation - a cross-sectional study in a pre-admission setting. F1000Res. 2015;4:123.

8. Paquissi FC. The role of inflammation in cardiovascular diseases: the predictive value of neutrophil-lymphocyte ratio as a marker in peripheral arterial disease. Ther Clin Risk Manag. 2016;12:851-860.

9. Guthrie GJ, Charles KA, Roxburgh CS, Horgan PG, McMillan DC, Clarke SJ. The systemic inflammation-based neutrophil-lymphocyte ratio: experience in patients with cancer. Crit Rev Oncol Hematol. 2013; 88(1):218-230.

10. Ethier JL, Desautels D, Templeton A, Shah PS, Amir E. Prognostic role of neutrophil-to-lymphocyte ratio in breast cancer: a systematic review and meta-analysis. Breast Cancer Res. 2017;19(1):2. 
11. Afari ME, Bhat T. Neutrophil to lymphocyte ratio (NLR) and cardiovascular diseases: an update. Expert Rev Cardiovasc Ther. 2016;14(5): 573-577.

12. Bhat T, Teli S, Rijal J, et al. Neutrophil to lymphocyte ratio and cardiovascular diseases: a review. Expert Rev Cardiovasc Ther. 2013;11(1): $55-59$.

13. Shao Q, Chen K, Rha SW, Lim HE, Li G, Liu T. Usefulness of neutrophil/ lymphocyte ratio as a predictor of atrial fibrillation: a meta-analysis. Arch Med Res. 2015;46(3):199-206.

14. Gibson PH, Cuthbertson BH, Croal BL, et al. Usefulness of neutrophil/lymphocyte ratio as predictor of new-onset atrial fibrillation after coronary artery bypass grafting. Am J Cardiol. 2010;105(2): 186-191.

15. Arbel Y, Finkelstein A, Halkin A, et al. Neutrophil/lymphocyte ratio is related to the severity of coronary artery disease and clinical outcome in patients undergoing angiography. Atherosclerosis. 2012;225(2): 456-460.

16. Bulow NMH, Colpo E, Duarte MF, et al. Inflammatory response in patients under coronary artery bypass grafting surgery and clinical implications: a review of the relevance of dexmedetomidine use. ISRN Anesth. 2014;2014:905238.

17. Núñez J, Núñez E, Bodí V, et al. Usefulness of the neutrophil to lymphocyte ratio in predicting long-term mortality in ST segment elevation myocardial infarction. Am J Cardiol. 2008;101(6):747-752.
18. Papa A, Emdin M, Passino C, Michelassi C, Battaglia D, Cocci F. Predictive value of elevated neutrophil-lymphocyte ratio on cardiac mortality in patients with stable coronary artery disease. Clin Chim Acta. 2008; 395(1-2):27-31.

19. Duffy BK, Gurm HS, Rajagopal V, Gupta R, Ellis SG, Bhatt DL. Usefulness of an elevated neutrophil to lymphocyte ratio in predicting long-term mortality after percutaneous coronary intervention. Am J Cardiol. 2006;97(7):993-996.

20. Gibson PH, Croal BL, Cuthbertson BH, et al. Preoperative neutrophillymphocyte ratio and outcome from coronary artery bypass grafting. Am Heart J. 2007;154(5):995-1002.

21. Gedikli O, Dogan A, Altuntas I, et al. Inflammatory markers according to types of atrial fibrillation. Int J Cardiol. 2007;120(2):193-197.

22. Danesh J, Collins R, Appleby P, Peto R. Association of fibrinogen, C-reactive protein, albumin, or leukocyte count with coronary heart disease: meta-analyses of prospective studies. JAMA. 1998;279(18):1477-1482.

23. Turkmen K, Guney I, Yerlikaya FH, Tonbul HZ. The relationship between neutrophil-to-lymphocyte ratio and inflammation in end-stage renal disease patients. Ren Fail. 2012;34(2):155-159.

24. Motomura T, Shirabe K, Mano Y, et al. Neutrophil-lymphocyte ratio reflects hepatocellular carcinoma recurrence after liver transplantation via inflammatory microenvironment. J Hepatol. 2013;58(1):58-64.

25. Edwards M, Whittle J, Ackland GL. Biomarkers to guide perioperative management. Postgrad Med J. 2011;87(1030):542-549.
Pragmatic and Observational Research

\section{Publish your work in this journal}

Pragmatic and Observational Research is an international, peer-reviewed, open access journal that publishes data from studies designed to reflect more closely medical interventions in real-world clinical practice compared with classical randomized controlled trials (RCTs). The manuscript management system is completely online and includes a very quick and fair peer-review

\section{Dovepress}

system. Visit http://www.dovepress.com/testimonials.php to read real quotes from published authors.

Submit your manuscript here: https://www.dovepress.com/pragmatic-and-observational-research-journal 